\title{
BMJ Open Scoping review protocol on non- pharmacological interventions for interpersonal and self-directed violence in adults with severe mental illness
}

\author{
Maria Concepcion Moreno-Calvete (D) , ${ }^{1}$ Ivan Ruiz-lbañez, ${ }^{2}$ \\ Jose Juan Uriarte-Uriarte ${ }^{1}$
}

To cite: Moreno-Calvete MC, Ruiz-lbañez I, Uriarte-Uriarte JJ. Scoping review protocol on nonpharmacological interventions for interpersonal and selfdirected violence in adults with severe mental illness. BMJ Open 2020;10:e037006. doi:10.1136/ bmjopen-2020-037006

- Prepublication history for this paper is available online. To view these files, please visit the journal online (http://dx.doi. org/10.1136/bmjopen-2020037006).

Received 15 January 2020 Revised 08 July 2020 Accepted 15 August 2020

\section{Check for updates}

\section{Author(s) (or their} employer(s)) 2020. Re-use permitted under CC BY-NC. No commercial re-use. See rights and permissions. Published by BMJ.

${ }^{1}$ Biocruces Bizkaia Health Research Institute, Basque Health Service, Bizkaia Mental Health Network, Bilbao, Biscay, Spain

${ }^{2}$ Basque Health Service, Bizkaia Mental Health Network, Assertive Community Treatment (ACT) Team, Buenavista Health Centre, Portugalete, Biscay, Spain

\section{Correspondence to}

Maria Concepcion Moreno-

Calvete;

mariaconcepcion.

morenocalvete@osakidetza.eus

\section{ABSTRACT}

Introduction Violence committed by people with mental illness has implications for mental health policy and clinical practice. Several strategies to reduce the risk of aggressive and violent behaviour have been proposed, and these include non-pharmacological interventions. There is, however, a need to identify which of these interventions are effective, and as a first step, we will conduct a scoping review to identify non-pharmacological interventions for self-directed or interpersonal violence in adults with severe mental illness across different conditions and settings.

Methods and analysis This is a scoping review protocol. The review will include any randomised controlled trials (RCTs) and cluster RCTs that assess the efficacy of interventions on self-directed or interpersonal violence with no restrictions on the control treatment in people with severe mental illness in any setting. No restrictions will be applied in terms of language or date of publication. To identify studies, a search will be performed in the following databases: Embase, MEDLINE (via PubMed), PsycINF0, CINAHL, LILACS, SciELO, Cochrane Library, Web of Science, Scopus, ProQuest, Epistemonikos and databases of clinical trials. The Preferred Reporting Items for Systematic Reviews and Meta-Analysis (PRISMA) statement will be followed for reporting the findings, including the use of a PRISMA flow diagram. A standardised form will be used to extract data from studies. The findings will be classified using conceptual categories that will be specified in detail and a descriptive summary of the main results will be created. Moreover, it will be assessed whether the studies identified have been included in systematic reviews or meta-analyses and the results will be used to generate a conceptual map.

Ethics and dissemination No patients or other participants will be involved in this study. We will prepare a manuscript for publication in a peer-reviewed journal and the results will be presented at mental health conferences.

\section{INTRODUCTION}

Individuals with severe mental illness "suffer from severe psychiatric disorders together with long-term mental disturbances, which entail a variable degree of disability and social dysfunction, and must be cared for by means
Strengths and limitations of this study

- This scoping review will contribute to identifying non-pharmacological interventions for self-directed and interpersonal violence in people with severe mental illness.

- This is a review-based approach to mapping the literature on a complex and broad topic.

- No restrictions will be applied in terms of language or date of publication.

- As a scoping review, this study will identify any nonpharmacological interventions but not assess the quality of the evidence.

- The small number of studies on this topic could be a limitation.

of different social and health resources of the psychiatric and social healthcare network". ${ }^{1}$ The conditions from the International Classification of Diseases, $10^{\text {th }}$ Revision (ICD-10) included in severe mental illness are: schizophrenic disorders, schizotypal disorders, persistent delusional disorders, induced delusional disorders, schizoaffective disorders, other non-organic psychotic disorders, bipolar disorder, major depressive episode with psychotic features, recurrent major depressive disorders and compulsive obsessive disorder. ${ }^{23}$ Evidence shows an association between mental illnesses, such as schizophrenia and related disorders, and increased rates of convictions for a violent offence, suicide and premature mortality. ${ }^{4}$

The WHO defines violence as "the intentional use of physical force or power, threatened or actual, against oneself, another person, or against a group or community, that either results in or has a high likelihood of resulting in injury, death, psychological harm, maldevelopment or deprivation" ${ }^{5}$ The relationship between severe mental illness and violent behaviour is more complex 
than it seems. ${ }^{6}$ In the USA, it has been found that while approximately $2 \%$ of the general population had perpetrated violence, the figure was as high as $7 \%$ to $8 \%$ among people with severe mental illness. ${ }^{7}$ Specifically, the prevalence rates of violence in psychiatric patients ranged from $2.3 \%$ to $13.0 \%$ in outpatients and from $16.0 \%$ to $23.0 \%$ during hospitalisation. ${ }^{8}$

On the other hand, a high level of mental disorders has been found among prisoners with convictions for violent or non-violent crimes. Some studies in prison populations have suggested that the prevalence of psychosis is $1.8 \%$ to $3.7 \%$ and of major depression is $11.4 \%$ to $14.7 \%$, while obsessive-compulsive disorder was diagnosed in $0.3 \% .^{9-11}$ Additionally, mental disorders have been associated with suicide risk and violence in prisoners. ${ }^{12} 13$

A systematic review revealed that over $96 \%$ of offenders with severe mental illness convicted of homicide had psychiatric symptoms at the time of the crime and several risk factors have been associated with violence in people with schizophrenia such as substance abuse, insight, impulsiveness, psychopathy, motor speed, a global measure of cognition, baseline injurious violence and medication non-adherence. ${ }^{14-16}$ In addition to this, violence is associated with a history of violent victimisation and recent violent victimisation, and hence, victimisation could contribute to violence. ${ }^{1617}$

Despite the relationship between victimisation and violence, and the prevalence of victimisation being higher than that of violence in people with severe mental illness, the focus of the society is often on the violence. ${ }^{18} 19 \mathrm{~A}$ study showed that the narratives about severe mental illness and violence increase the stigma, and the public is frequently exposed to portrayals of severe mental illness and violence in news media. ${ }^{2021}$

Further, the lifetime suicide risk for people with schizophrenia is estimated at about $4.9 \% .^{22}$ Risk factors implicated in suicide in people with schizophrenia include affective symptoms, history of a suicide attempt and number of psychiatric admissions, younger age, closeness to illness onset, older age at illness onset, male sex and substance abuse, while the period during or following psychiatric discharge has been identified as a high-risk time. ${ }^{23}$ Notwithstanding that suicide and interpersonal violence are not the same phenomena, they are observed together in people with schizophrenia, and offenders with psychosis who were at risk of suicide and violence had a history of more suicide attempts and more convictions for violent offences. ${ }^{24} 25$

Several studies on pharmacological and nonpharmacological interventions have been found but there is heterogeneity in interventions, and no scoping review identifying the evidence on these interventions has been reported. Concerning pharmacological treatments, studies on interpersonal violence have focussed on treatments such as long-acting injectable antipsychotics, antipsychotic polypharmacy, high-dose monotherapy or antipsychotic dosage and studies on pharmacological interventions for self-directed violence have been related to treatments such as clozapine, lithium or anticonvulsants. $^{26-31}$

Regarding non-pharmacological interventions on interpersonal violence, several studies have evaluated interventions in various settings such as structured short-term risk assessment, cognitive behavioural therapy, involuntary outpatient commitment, a community-based integrated intervention, integrated treatment plus assertive community treatment and metacognition-oriented social skills training. ${ }^{32-37}$ On the other hand, several studies have assessed non-pharmacological interventions on selfdirected violence including cognitive-behavioural suicide prevention, 'housing first', a cognitive behavioural therapy worry-reduction intervention and psychoeducation. ${ }^{38-41}$

A systematic review was conducted related to nonpharmacological interventions on interpersonal violence in people with severe mental illness; ${ }^{42}$ however, it did not include all diagnoses of severe mental illness, the search was limited in terms of language and databases and the search period was until June 2015, and hence, it does not consider more recently published studies such as those of Kang et al, Inchausti et al, Kingston et al. ${ }^{35} 43$ Additionally, an umbrella review was performed of interventions in general and forensic psychiatry on violence prevention. ${ }^{44}$ Nonetheless, this review is not focussed on severe mental illness and intervention studies included nonrandomised as well as randomised controlled designs. No similar systematic reviews have been found related to non-pharmacological interventions on self-directed violence in the context of interest.

The intervention strategies for interpersonal and selfdirected violence in people with severe mental illness vary across studies. In this situation, that is, when the body of knowledge is heterogeneous, scoping reviews allow assessment of the extent of the evidence on a topic and the value of conducting a systematic review or an overview of systematic reviews to summarise findings. ${ }^{45}$

\section{Rationale}

The association between mental illness and interpersonal or self-directed violence has significance from a public health perspective and implications for mental health policy and clinical practice. ${ }^{6} 86$ Interpersonal violence is the factor with the most negative influence on the general population's view of mental illness and one of the main contributors to the associated stigma. ${ }^{6}$ Denial of this association is not the best way for mental health practitioners to deal with the problem and hinders the setting up interventions for preventing and tackling violence in this context. Furthermore, the role of mental illness in suicide requires an effort to strengthen mental healthcare to prevent suicide in this population. ${ }^{47}$

A combination of pharmacological and nonpharmacological treatment is necessary to manage interpersonal and self-directed violence in people with severe mental illness.

This review will be focussed on non-pharmacological interventions for interpersonal and self-directed violence 
in people with severe mental illness in any setting. The complexity and heterogeneity of non-pharmacological interventions on interpersonal and self-directed violence for people with severe mental illness, the existence of new evidence, the relevance of this topic and the absence of a scoping review warrant this study as the first step to decide whether it is necessary to conduct a systematic review or an overview of systematic reviews on this topic.

This study may be of interest to mental health practitioners and in particular might help in clinical practice by increasing the knowledge of strategies for interpersonal and self-directed violence in this population. Regarding future research, this scoping review will describe current research and provide information for designing additional studies or related projects such as a systematic review or overview. In the case of a lack of evidence, the information could stimulate research on this topic. On the other hand, in the case of finding RCTs and cluster RCTs included in systematic reviews, it would suggest the need to carry out an overview to synthesise the evidence from the systematic reviews while the absence of systematic reviews would suggest the need for a systematic review on this topic.

\section{Objectives}

This study aims to identify and describe nonpharmacological interventions for self-directed or interpersonal violence in people with serious mental illness in any setting.

Additionally, we will investigate whether each intervention found has been included in any systematic reviews or meta-analyses to decide whether it is necessary to conduct a systematic review or an overview of systematic reviews on this topic.

\section{Review questions}

What kinds of non-pharmacological interventions for selfdirected or interpersonal violence in people with severe mental illness have been studied in any setting?

What are the durations, frequencies, settings and timings of non-pharmacological interventions for selfdirected or interpersonal violence in people with severe mental illness?

What are the effects of these non-pharmacological interventions for self-directed or interpersonal violence described in the literature?

Which studies have been included in a systematic review/meta-analysis?

\section{METHODOLOGY}

\section{Protocol design and registration}

This protocol was developed considering the Preferred Reporting Items for Systematic Reviews and Meta-Analysis Protocols (PRISMA-P) 2015 guidelines, ${ }^{48}$ with recommended material being included as indicated in online supplemental file 1. It has been registered with the Open Science Framework (https://osf.io/d56a2/).

\section{Eligibility criteria}

Studies will be selected using population, concept and context criteria:

\section{Population}

Inclusion criteria: adults with a diagnosis of severe mental illness or any condition included in severe mental illness: ${ }^{2}$ schizophrenic disorder, schizoaffective disorder, schizotypal disorder, persistent or induced delusional disorder, bipolar disorder, obsessive-compulsive disorder, major depressive episode with psychotic features, recurrent major depressive disorders, atypical psychosis or other non-organic psychosis (according to international classification systems: Diagnostic and Statistical Manual of Mental Disorders or International Classification of Diseases). The criteria established for severe mental illness are in accordance with clinical practice guidelines on psychosocial interventions in severe mental illness ${ }^{2}$ and eligible methods for identifying the population include clinical diagnosis and research interview, among others. We will also include studies in adults with severe mental illness and substance abuse. No restrictions will be placed on the setting.

Exclusion criteria: people with substance abuse alone or with organic mental disorders, dementia, mental retardation or learning disabilities.

\section{Concept}

The study will consider non-pharmacological interventions in combination or not with another intervention for interpersonal or self-directed violence in people with severe mental illness. Violence should be established in accordance with the WHO definition ${ }^{5}$ and may include physical or verbal aggression, physical or verbal threats, psychological or emotional abuse, criminal activity, property damage, suicide attempt, completed suicide or self-harm.

There will be no restrictions regarding the duration, frequency, provider or timing of the interventions. Interventions may be:

- Direct: if they affect patients.

- Indirect: if their influence is not on patients, but is to their benefit.

- Targeting family, professionals or the community or at a public health level.

Concomitant pharmacological interventions will be allowed, if they are administered in the same way in all study groups (intervention and control groups).

Exclusion criteria: Instrumental treatments or brain stimulation treatments such as electroconvulsive therapy, transcranial direct current stimulation or transcranial magnetic stimulation.

Comparisons of interest: All interventions included will need a comparison group. The control treatment may be another treatment, placebo or treatment as usual for interpersonal or self-directed violence ("treatment as usual' being defined as the normal level of care provided in the area in which the study has been performed). 
The outcomes of interest are:

\section{Main outcomes}

Violent behaviour

- Interpersonal violence: Physical or verbal aggression, physical or verbal threats, psychological or emotional abuse, criminal activity or property damage.

- Self-directed violence: Self-harm, suicide attempt or completed suicide.

\section{Additional outcomes}

- Agitation behaviour.

- Substance use: Drug use or alcohol use.

- Illness severity:

- Psychiatric symptoms.

- Functioning.

- Use of physical restraints or seclusion.

- Use of medication in an emergency.

The studies should include violent behaviour (interpersonal or self-directed violence) as an outcome to be included in the scoping review.

\section{Context elements}

This study will include any RCTs or cluster RCTs that assess the efficacy of non-pharmacological interventions on interpersonal or self-directed violence in people with severe mental illness in any setting. No restrictions will be applied on the control treatment (another treatment, placebo or treatment as usual) or in terms of language or date of publication.

\section{Information sources and search strategy}

The primary systematic literature searches will be conducted using a carefully designed search strategy. The combinations of keywords and search terms will be adapted for use with other databases in combination with database-specific filters, where these are available. The following electronic databases will be searched: Embase, MEDLINE (via PubMed), PsycINFO, CINAHL, LILACS, SciELO, Cochrane Library (the Cochrane Central Register of Controlled Trials(CENTRAL) and Cochrane Database of Systematic Reviews), Web of Science, Scopus, ProQuest, Epistemonikos and clinical trial databases ( www.controlled-trials.com, www.ClinicalTrials.gov and the WHO International Clinical Trials Registry Platform), among others. Further, the studies included in the clinical practice guidelines of various national health systems and the references of studies included will be reviewed to identify additional articles of interest. RefWorks reference management software will be used to manage the articles. $^{49}$

As an example, the search strategy to be used on Web of Science is:

TI=(schizophrenia OR schizophren* OR psychotic disorders OR psycho* OR schizoaffective OR schizotypal personality disorder OR schizotyp* OR delirium OR severe mental illness OR severe mental disorder OR mental disorders OR bipolar and related disorders OR bipolar disorder* OR manic-depressive OR manic depressive OR bipolar affective OR mania* OR manic disorder OR obsessive-compulsive disorder OR obsessivecompulsive OR anankastic OR depressive disorder, major OR major depressi* OR diagnosis, dual (psychiatry) OR psychiatric dual OR dual diagnosis) $\mathrm{AND} \mathrm{TI}=$ (violence OR violen* OR aggression OR aggressi* OR damage OR prison* OR offens* OR suicid* OR self-harm OR self-injur*) AND TI $=$ (randomized controlled trial OR controlled clinical trial OR randomized OR placebo OR randomly OR trial).

Further, a search will be performed to determine whether or not the RCTs and cluster RCTs selected for the scoping review have been included in systematic reviews. The information gathered from this process will contribute to the assessment of whether what is required subsequently is a systematic review, analysing data from primary studies which have not previously been included in any systematic reviews or an overview of systematic reviews, if numerous such reviews have already been carried out.

\section{Study selection and data extraction process}

The titles and/or abstracts of studies retrieved using the search strategy, and those from other sources, will be screened independently by two review authors (MCM and IR) to identify studies that potentially meet the inclusion criteria outlined above. Full texts of potentially eligible studies will then be retrieved and independently assessed for inclusion/exclusion by the same two authors, and any disagreements will be resolved through discussion and consensus. A standardised form will be used to extract data from the studies identified for final inclusion in the review.

All relevant data will be included to answer the scoping review questions. The information to be charted during the process will include: authors; publication year; country; study design; study population; settings; aim; details of the intervention and the control conditions; outcomes and times of measurement; findings and other relevant information. Two review authors (MCM and JJU) will extract the data independently; any discrepancies will be resolved through discussion and consensus. Any data found to be missing in the report of the studies, such as information about outcomes of interest or the population or any other information relevant to the scoping review will be requested directly from the study authors.

According to the scoping review's methodology, the aim is to identify and describe the evidence; therefore, the quality of individual studies will not be assessed.

\section{Data synthesis and reporting of findings}

A descriptive summary of the main results will be reported. The results will be classified under conceptual categories such as author(s), year of publication, place or country where the study was conducted, study design, aim, study population, setting, description of the intervention and the control conditions, outcomes and details of key findings. Intervention subgroups will be assessed 
and a conceptual map will be generated with the findings. A PRISMA flow diagram will be created to illustrate the progress of studies through the selection process and screening (indicating the results from the search, removal of duplicate citations, and so on). ${ }^{45}$

\section{Limitations}

Despite this being a scoping review on a complex and broad topic, violence is a very specific topic and we may find only a very small number of the studies. To maximise the number of publications found, several databases will be searched and no restrictions will be applied in terms of language or date of publication.

Another potential limitation is that the quality of the evidence will not be assessed. This is because it is to be a scoping review, the objective of which is not to conduct a critical appraisal but rather to identify and describe nonpharmacological interventions. Nonetheless, this scoping review will describe current research and provide information on which to base future research, especially related work such as a systematic review or an overview. Indeed, it will provide information to help decide whether to conduct a systematic review or an overview.

\section{Patient and public involvement}

No patients or other participants will be involved in this study.

\section{ETHICS AND DISSEMINATION}

This study does not require ethics approval. This scoping review will contribute to improve our understanding of violent behaviour in people with severe mental illness. A manuscript for publication in a peer-reviewed journal will be prepared and the results will be presented at mental health conferences.

Contributors MCM was the major contributor in writing the manuscript. JJU and IR critically revised the content and contributed to the manuscript. All authors gave their final approval of the version to be published.

Funding This work was supported by the Department of Health of the Government of the Basque Country, Spain (Grant number 2017111101). The funder has had no involvement in any aspect of the protocol or the decision to submit for publication and will not be involved in the study.

Competing interests None declared.

Patient consent for publication Not required.

Provenance and peer review Not commissioned; externally peer reviewed.

Open access This is an open access article distributed in accordance with the Creative Commons Attribution Non Commercial (CC BY-NC 4.0) license, which permits others to distribute, remix, adapt, build upon this work non-commercially, and license their derivative works on different terms, provided the original work is properly cited, appropriate credit is given, any changes made indicated, and the use is non-commercial. See: http://creativecommons.org/licenses/by-nc/4.0/.

\section{ORCID iD}

Maria Concepcion Moreno-Calvete http://orcid.org/0000-0001-9712-2319
REFERENCES

1 National Institute of Mental Health. Towards a model for a comprehensive community based mental health system. Washington DC: NIMH, 1987.

2 Guideline development group of the clinical practice guideline on psychosocial interventions in severe mental illness. clinical practice guideline on psychosocial interventions in severe mental illness. quality plan for the National health system, Ministry of health and social policy. Aragon health sciences Institute $-I+C S$; 2009. clinical practice guidelines in the Spanish NHS: I+CS no 2007/05.

3 World Health Organization. Icd-10: international statistical classification of diseases and related health problems: tenth revision. World Health organization, 2019. Available: https://icd.who.int/ browse10/2019/en

4 Fazel S, Wolf A, Palm C, et al. Violent crime, suicide, and premature mortality in patients with schizophrenia and related disorders: a 38-year total population study in Sweden. Lancet Psychiatry 2014;1:44-54.

5 Butchart A, Mikton C, Dahlberg LL, et al. Global status report on violence prevention 2014. Inj Prev 2015;21:213.

6 Varshney M, Mahapatra A, Krishnan V, et al. Violence and mental illness: what is the true story? J Epidemiol Community Health 2016;70:223-5.

7 Choe JY, Teplin LA, Abram KM. Perpetration of violence, violent victimization, and severe mental illness: balancing public health concerns. Psychiatr Serv 2008;59:153-64.

8 Elbogen EB, Johnson SC. The intricate link between violence and mental disorder: results from the National epidemiologic survey on alcohol and related conditions. Arch Gen Psychiatry 2009;66:152.

9 Fazel S, Seewald K. Severe mental illness in 33,588 prisoners worldwide: systematic review and meta-regression analysis. $\mathrm{Br} J$ Psychiatry 2012;200:364-73.

10 Zhong S, Zhu X, Chen Y, et al. High psychiatric morbidity and comorbidity among female prisoners in Hunan, China. Front Psychiatry 2020;11:271.

11 Huang $\mathrm{Y}$, Wang $\mathrm{Y}$, Wang $\mathrm{H}$, et al. Prevalence of mental disorders in China: a cross-sectional epidemiological study. Lancet Psychiatry 2019;6:211-24.

12 Zhong S, Zhu X, Mellsop G, et al. Mental health problems, history of drug use, and violent offending are associated with increased suicide risk in imprisoned females. Front Psychiatry 2019;10:395.

13 Walters GD. Criminal thinking as a mediator of the mental illnessprison violence relationship: a path analytic study and causal mediation analysis. Psychol Serv 2011;8:189-99.

14 Minero VA, Barker E, Bedford R. Method of homicide and severe mental illness: a systematic review. Aggress Violent Behav 2017;37:52-62.

15 Rund BR. A review of factors associated with severe violence in schizophrenia. Nord J Psychiatry 2018;72:561-71.

16 Buchanan A, Sint K, Swanson J, et al. Correlates of future violence in people being treated for schizophrenia. Am J Psychiatry 2019;176:694-701.

17 Witt K, van Dorn R, Fazel S. Risk factors for violence in psychosis: systematic review and meta-regression analysis of 110 studies. PLoS One 2013;8:e55942.

18 Tsigebrhan R, Shibre T, Medhin G, et al. Violence and violent victimization in people with severe mental illness in a rural lowincome country setting: a comparative cross-sectional community study. Schizophr Res 2014;152:275-82.

19 Severe mental disorders and the risk of violence: pathways through care and effective treatment strategies. European Commission Health and Food Safety Directorate-General. (Ref. Ares2016) 6133940. [Internet]. Available: https://ec.europa.eu/research/ participants/data/ref/other_eu_prog/other/hp/call-fiche/hp-call-fichepp2-3_en.pdf

20 McGinty EE, Webster DW, Jarlenski M, et al. News media framing of serious mental illness and gun violence in the United States, 19972012. Am J Public Health 2014;104:406-13.

21 McGinty EE, Goldman HH, Pescosolido BA, et al. Communicating about mental illness and violence: balancing stigma and increased support for services. J Health Polit Policy Law 2018;43:185-228.

22 Palmer BA, Pankratz VS, Bostwick JM. The lifetime risk of suicide in schizophrenia: a reexamination. Arch Gen Psychiatry 2005;62:247.

23 Popovic D, Benabarre A, Crespo JM, et al. Risk factors for suicide in schizophrenia: systematic review and clinical recommendations. Acta Psychiatr Scand 2014;130:418-26.

24 Witt K, Hawton K, Fazel S. The relationship between suicide and violence in schizophrenia: analysis of the clinical antipsychotic trials of intervention effectiveness (CATIE) dataset. Schizophr Res 2014;154:61-7. 
25 SanSegundo MS, Ferrer-Cascales R, Bellido JH, et al. Prediction of violence, suicide behaviors and suicide ideation in a sample of institutionalized offenders with schizophrenia and other psychosis. Front Psychol 2018:9:1385

26 Mohr P, Knytl P, Voráčková V, et al. Long-acting injectable antipsychotics for prevention and management of violent behaviour in psychotic patients. Int J Clin Pract 2017;71:e12997.

27 Morrissette DA, Stahl SM. Treating the violent patient with psychosis or impulsivity utilizing antipsychotic polypharmacy and high-dose monotherapy. CNS Spectr 2014;19:439-48.

28 Swanson JW, Swartz MS, Van Dorn RA, et al. Comparison of antipsychotic medication effects on reducing violence in people with schizophrenia. Br J Psychiatry 2008;193:37-43.

29 Tasmim S, Kolla NJ, Dada O, et al. Correlation between violence and antipsychotic dosage in schizophrenia: a secondary analysis of the clinical antipsychotic trials for intervention effectiveness (CATIE) dataset. Pharmacopsychiatry 2019;52:217-21.

30 Meltzer HY, Alphs L, Green Al, et al. Clozapine treatment for suicidality in schizophrenia: international suicide prevention trial (InterSePT). Arch Gen Psychiatry 2003;60:82.

31 Schaffer A, Isometsä ET, Tondo L, et al. Epidemiology, neurobiology and pharmacological interventions related to suicide deaths and suicide attempts in bipolar disorder: Part I of a report of the International Society for bipolar disorders Task force on suicide in bipolar disorder. Aust N Z J Psychiatry 2015;49:785-802.

32 Abderhalden C, Needham I, Dassen T, et al. Structured risk assessment and violence in acute psychiatric wards: randomised controlled trial. Br J Psychiatry 2008;193:44-50.

33 Haddock G, Barrowclough C, Shaw JJ, et al. Cognitive-behavioural therapy $v$. social activity therapy for people with psychosis and a history of violence: randomised controlled trial. Br J Psychiatry 2009;194:152-7.

34 Swanson JW, Swartz MS, Borum R, et al. Involuntary out-patient commitment and reduction of violent behaviour in persons with severe mental illness. Br J Psychiatry 2000;176:324-31.

35 Kang R, Wu Y, Li Z, et al. Effect of community-based social skills training and tai-chi exercise on outcomes in patients with chronic schizophrenia: a randomized, one-year study. Psychopathology 2016;49:345-55.

36 Calsyn RJ, Yonker RD, Lemming MR, et al. Impact of assertive community treatment and client characteristics on criminal justice outcomes in dual disorder homeless individuals. Crim Behav Ment Health 2005;15:236-48.
37 Inchausti F, García-Poveda NV, Ballesteros-Prados A, et al. The effects of metacognition-oriented social skills training on psychosocial outcome in schizophrenia-spectrum disorders: a randomized controlled trial. Schizophr Bull 2018;44:1235-44.

38 Haddock G, Pratt D, Gooding PA, et al. Feasibility and acceptability of suicide prevention therapy on acute psychiatric wards: randomised controlled trial. BJPsych Open 2019;5:e14.

39 Aquin JP, Roos LE, Distasio J, et al. Effect of housing first on suicidal behaviour: a randomised controlled trial of homeless adults with mental disorders. Can J Psychiatry 2017;62:473-81.

40 Freeman D, Dunn G, Startup H, et al. Effects of cognitive behaviour therapy for worry on persecutory delusions in patients with psychosis (wit): a parallel, single-blind, randomised controlled trial with a mediation analysis. Lancet Psychiatry 2015;2:305-13.

41 Rabovsky K, Trombini M, Allemann D, et al. Efficacy of bifocal diagnosis-independent group psychoeducation in severe psychiatric disorders: results from a randomized controlled trial. Eur Arch Psychiatry Clin Neurosci 2012;262:431-40.

42 Rampling J, Furtado V, Winsper C, et al. Non-Pharmacological interventions for reducing aggression and violence in serious mental illness: a systematic review and narrative synthesis. Eur Psychiatry 2016;34:17-28

43 Kingston DA, Olver ME, McDonald J, et al. A randomised controlled trial of a cognitive skills programme for offenders with mental illness. Crim Behav Ment Health 2018;28:369-82.

44 Wolf $A$, Whiting $D$, Fazel S. Violence prevention in psychiatry: an umbrella review of interventions in general and forensic psychiatry. $J$ Forens Psychiatry Psychol 2017;28:659-73.

45 Tricco AC, Lillie E, Zarin W, et al. PRISMA extension for scoping reviews (PRISMA-ScR): checklist and explanation. Ann Intern Med 2018;169:467.

46 Brådvik L. Suicide risk and mental disorders. Int J Environ Res Public Health 2018;15:2028.

47 Too LS, Spittal MJ, Bugeja L, et al. The association between mental disorders and suicide: a systematic review and meta-analysis of record linkage studies. J Affect Disord 2019;259:302-13.

48 Shamseer L, Moher D, Clarke M, et al. Preferred reporting items for systematic review and meta-analysis protocols (PRISMA-P) 2015 elaboration and explanation. BMJ 2015;349:g7647.

49 RefWorks. ProQuest, 789 East Eisenhower Parkway, Ann Arbor, MI 48108. Available: http://refworks.proquest.com 